\title{
RESEARCH
}

Open Access

\section{The specific ex vivo released cytokine profile is associated with ischemic stroke outcome and improves its prediction}

\author{
Elzbieta Klimiec-Moskal ', Marcin Piechota², Joanna Pera' ${ }^{1}$, Kazimierz Weglarczyk ${ }^{3}$, Agnieszka Slowik', \\ Maciej Siedlar ${ }^{3}$ and Tomasz Dziedzic ${ }^{1^{*}}$ (1)
}

\begin{abstract}
Background: Inflammation is associated with poor outcome after stroke. A relationship between ex vivo cytokine synthesis and stroke outcome remains unclear. We explored an association between ex vivo cytokine release, circulating interleukin (IL)- 6 as a marker of systemic inflammation, and stroke prognosis. We assessed the utility of ex vivo synthesized cytokines for predicting stroke outcome.

Methods: We collected blood from 248 ischemic stroke patients and stimulated it ex vivo with lipopolysaccharide. We measured concentration of synthesized cytokines (TNFa, IP-10, IL-13, IL-6, IL-8, IL-10, and IL-12) and plasma IL-6. We assessed functional outcome 3 months after stroke using the modified Rankin Scale. To assess the prognostic ability of cytokines, we applied multivariate logistic regression, cluster analysis, and construction of multimarker score.

Results: Decreased release of IP-10, TNFa, IL-1 3 , and IL-12; increased release of IL-10 and IL-8; and higher plasma IL6 level were associated with poor outcome. Cluster analysis identified three groups of patients with distinct cytokine profiles. The group with the worst outcome demonstrated high synthesis of IL-10, IL-8, IL-1 $\beta$, and IL-6 and low synthesis of IL-12, IP-10, and TNFa accompanied by high circulating IL-6 level. The group with the best prognosis showed high synthesis of TNFa, IP-10, IL-12, IL-1 $\beta$, and IL-6; low synthesis of IL-10 and IL-8; and low plasma IL-6. Patients with intermediate outcome had low synthesis of all cytokines accompanied by low circulating IL-6. We constructed a multimarker score composed of ex vivo released IL-12, IL-10, TNFa, and plasma IL-6. Addition of this score to clinical variables led to significant increase in c-statistic $(0.81$ vs $0.73, p=0.02)$ and net reclassification improvement.
\end{abstract}

Conclusion: The decreased ex vivo release of pro-inflammatory cytokines and increased release of IL-10 and IL-8 are related to poor outcome after stroke. Cytokine-based multimarker score adds prognostic value to clinical model for predicting stroke outcome.

Keywords: Stroke, Cytokine, Outcome, Inflammation, Biomarker, Prediction

\footnotetext{
*Correspondence: dziedzic@cm-uj.krakow.pl

'Department of Neurology, Jagiellonian University Medical College, ul.

Botaniczna 3, 31-503 Kraków, Poland

Full list of author information is available at the end of the article
}

(c) The Author(s). 2020 Open Access This article is distributed under the terms of the Creative Commons Attribution 4.0 International License (http://creativecommons.org/licenses/by/4.0/), which permits unrestricted use, distribution, and reproduction in any medium, provided you give appropriate credit to the original author(s) and the source, provide a link to the Creative Commons license, and indicate if changes were made. The Creative Commons Public Domain Dedication waiver (http://creativecommons.org/publicdomain/zero/1.0/) applies to the data made available in this article, unless otherwise stated. 


\section{Background}

Acute ischemic stroke is accompanied by both immunodepression and systemic inflammation [1, 2]. Post-stroke immunodepression refers to suppression of both innate and adaptive immune response and is marked by transient lymphopenia, functional deactivation of monocytes, and splenic atrophy. At the cytokine level, immunodepression is reflected in humans by the reduced ex vivo tumor necrosis factor alpha (TNFo) synthesis after whole blood or monocyte stimulation with endotoxin $[3,4]$ whereas circulating interleukin-6 (IL-6) is the main cytokine related to systemic inflammation [5].

Numerous studies showed that elevated blood level of IL6 is an independent predictor of poor functional outcome after stroke $[6,7]$. However, the addition of IL-6 to a validated, clinical-based stroke prognostic model did not improve the prediction of poor outcome. Much less is known about the prognostic significance of ex vivo cytokine production in stroke patients. Few studies suggest that the reduced ex vivo TNF $\alpha$ and interferon-gamma-inducible protein 10 (IP-10) synthesis after endotoxin challenge is associated with poor functional outcome after stroke $[8,9]$.

A better understanding of interactions between cytokine synthesis and stroke prognosis is important for at least two reasons. First, cytokine synthesis could be a potential therapeutic target in acute stroke [10]. Second, individual cytokines released by blood cells or their combination might serve as a biomarker to stratify risk of unfavorable outcome after stroke.

In this study, we aimed to explore an association between ex vivo cytokine synthesis, circulating IL-6 as a marker of systemic inflammation and stroke prognosis. We also examined the individual and collective utility of ex vivo synthesized cytokines for predicting stroke outcome.

\section{Methods}

\section{Participant and clinical assessment}

We prospectively recruited participants from consecutive stroke patients admitted to the Department of Neurology, University Hospital in Krakow, Poland, between October 2016 and September 2018. The inclusion criteria were (1) ischemic stroke, (2) admission within $24 \mathrm{~h}$ from symptom onset, (3) pre-stroke modified Rankin Scale (mRS) score 02, (4) National Institute of Health Stroke Scale (NIHSS) score on admission $>3$, and (5) informed consent. We excluded patients with chronic inflammatory, autoimmune, or cancerous diseases. Bioethics Committee of Jagiellonian University approved the study protocol (decision number: 122.6120.249.2016), and each patient gave an informed consent.

For ischemic stroke diagnosis, we used the updated definition from the American Heart Association/American Stroke Association [11]. Each patient underwent the head CT scan on admission. To assess stroke severity on admission, we used NIHSS score [12]. We used the TOAST criteria to determine stroke etiology [13]. We evaluated functional outcome 3 months after stroke with the mRS [14]. We defined poor outcome as a mRS score 3-6.

\section{Laboratory assays}

We collected venous blood in heparinised tubes (Sarstedt, Germany) at day 3 after stroke onset. To avoid diurnal variation in cytokine production, we took blood between 7.00 and $7.30 \mathrm{am}$. Subsequently, we diluted the whole blood by 1:5 in sterile RPMI 1640 medium supplemented with L-glutamine (Sigma Aldrich, St. Louis, MO, USA) and we stimulated it in sterile tubes (Lonza, Walkersville, MD, USA) with lipopolysaccharide (LPS $10 \mathrm{ng} / \mathrm{mL}$, E. coli 0111:B4, Sigma Aldrich, St. Louis, MO, USA) at $37{ }^{\circ} \mathrm{C}$ and $5 \% \mathrm{CO} 2$. Based on previous publications $[9,15]$, we stimulated blood for $4 \mathrm{~h}$ for TNF $\alpha$ and IP-10 and $24 \mathrm{~h}$ for interleukin IL-1 $\beta$, IL-6, IL-8, IL-10, and IL-12p70. We removed supernatants and stored them at $-80^{\circ} \mathrm{C}$.

To measure concentrations of TNFa and IP-10, we used commercially available ELISA kits from R\&D Systems (Minneapolis, MN, USA). We measured IL-1 $\beta$, IL6, IL-8, IL-10, and IL-12p70 concentration using a cytometric bead array immunoassay (Human Inflammatory Kit, BD Bioscience, San Diego, CA, USA). To measure the level of IL-6 in plasma, we used ELISA kits from R\&D Systems (Minneapolis, MN, USA) with a detection limit of $0.11 \mathrm{pg} / \mathrm{ml}$.

\section{Statistical analyses}

To better understand an association between cytokines and the poor outcome, we aimed to implement different statistical approaches.

As a first step, we compared baseline characteristics and cytokine levels between the group with good outcome and the group with poor outcome using $x^{2}$ test for proportions and Mann-Whitney $U$ test for continuous variables. To find independent predictors of outcome, we performed logistic regression analyses. We standardized cytokines to the same scale $($ mean $=0, S D=1)$ to facilitate comparison between them. We used univariate analysis to determine clinical and laboratory predictors. Then, cytokines with $p<0.05$ in the univariate regression were assessed individually in backward elimination models adjusted for significant clinical predictors. We used a retention value of $p<0.05$. Finally, we performed a backward elimination model containing all cytokines significant in the univariate analysis adjusted for clinical predictors.

In the second step, we wanted to divide subjects into a small number of groups with different cytokine concentrations and test whether any specific profile could be 
related to the poor outcome. Before clustering, we log transform cytokines because they were right-skewed and then we standardize them to the same scale (mean $=0$, $\mathrm{SD}=1)$. To check collinearity between cytokines, we calculated Pearson's correlation coefficient and Spearman's rho using a threshold of 0.7 as an indicator of high collinearity [16]. We grouped patients using agglomerative hierarchical clustering with Ward's method of minimum variance and the squared Euclidean distance metric [17]. We considered this method appropriate for our purposes as it could construct relatively compact clusters with emphasis on differences between observations [18]. Clustering was unsupervised and blinded to outcome. We determined the optimal number of clusters based on 30 indices provided by NbClust package in $\mathrm{R}$ [19]. To better characterize cytokine profiles, we compared clusters with Kruskal-Wallis test followed by post-hoc Dunn's test. Next, to determine an association between clusters and the outcome, we performed uni- and multivariate logistic regressions. The latter was adjusted for previously identified clinical predictors.

In the final step, we constructed the multimarker score to further evaluate the prognostic value of investigated cytokines. We included cytokines that remained significant in the final backward elimination model. The score was defined as:

$$
\begin{aligned}
\text { Score }= & \left(\beta_{\mathrm{A}} \times \text { cytokine } \mathrm{A}\right)+\left(\beta_{\mathrm{B}} \times \text { cytokineB }\right) \\
& +\left(\beta_{\mathrm{C}} \times \text { cytokineC }\right), \text { etc. },
\end{aligned}
$$

where $\beta_{A}, \beta_{B}$, and $\beta_{C}$ stand for regression $\beta$ coefficients for cytokines $A, B$, and $C$, respectively, from the backward regression model. We used a model with two most important predictors of outcome: stroke severity on admission and age as the best-fit clinical model [20]. Then, we compared the sole best-fit clinical model with the best-fit clinical model containing the multimarker score. We also compared the best-fit clinical model containing the multimarker score with best-fit clinical models containing individual cytokines. To evaluate the ability of models to discriminate between good and poor outcome, we calculated areas under receiver operator curves (AUC). An AUC of 1 indicates perfect discrimination and 0.5 no discrimination. We also assessed calibration with the Hosmer-Lemeshow $\chi^{2}$ statistic. Then, we calculated the integrated discrimination improvement (IDI), a measure of the model to improve average sensitivity without reducing average specificity [21]. To assess the ability of the score to reclassify risk, we calculated net reclassification improvement (NRI) metric [21]. Similarly to the previous study [22], we used clinically relevant thresholds $(<10 \%, 10-50 \%, 50-90 \%,>90 \%)$ for the predicted probability of the poor outcome. The thresholds are based on the assumption that one would need to be very certain of a good or poor outcome before avoiding treatment such as thrombolysis or selecting patients for palliative care only. We also calculated a continuous NRI [23].

Statistical analyses were performed in STATA version 14 (StataCorp, College Station, TX) and packages "NbClust" [17] and "PredictABEL" [24] in R Statistical Software (Foundation for Statistical Computing, Vienna, Austria).

\section{Results}

\section{Patients characteristic}

We recruited 250 patients into the study. Information on 3-month functional status was available for 248 patients, and they were included into further analyses (median age 69 years, IQs $61-79 ; 41.1 \%$ female; median NIHSS score 9, IQs 5-17). From the final cohort, 111 participants (44.8\%) had a poor 3-month functional outcome. Table 1 shows comparisons of baseline characteristics and cytokine levels between the group with good outcome and the group with poor outcome.

Patients with poor outcome were older and had higher NIHSS score on admission. They had the increased release of IL-10 and IL- 8 and the decreased release of IL12 p70, IL-1 $\beta$, TNF $\alpha$, and IP-10 after ex vivo stimulation compared to the group with good outcome. Plasma level of IL-6 was also higher in the patients with worse outcome.

In the univariate analysis, older age and higher NIHSS score on admission were only clinical predictors of poor outcome (Table 2). Among cytokines, higher release of IL-10 and IL-8 after ex vivo stimulation as well as higher plasma level of IL- 6 was associated with worse outcome. In contrast, the higher release of IL-12p70, IL-1 $\beta$, TNF $\alpha$, and IP-10 was related to the decreased risk of poor outcome. These results remained significant after adjustment for age and stroke severity. However, when we forced all cytokines into the backward elimination model adjusted for clinical predictors, only IL-12p70, IL-10, TNF $\alpha$, and plasma IL- 6 levels remained independent laboratory predictors of outcome.

\section{Cluster analysis}

We included plasma level of IL- 6 and the following ex vivo produced cytokines: IL-12p70, IL-10, IL-6, IL-8, IL-1 $\beta$, TNF $\alpha$, and IP-10 into cluster analysis. Pearson's correlation coefficients or Spearman's rho between included cytokines did not exceed the threshold of 0.7.

We compared different numbers of clusters (maximal number of 6) based on 30 indices provided by NbClust package. The three-cluster solution showed the best 
Table 1 Comparison of baseline characteristics and levels of cytokines between good and poor outcome groups

\begin{tabular}{|c|c|c|c|}
\hline & $\begin{array}{l}\text { Good outcome (mRS 0-2) } \\
n=137\end{array}$ & $\begin{array}{l}\text { Poor outcome (mRS 3-6) } \\
n=111\end{array}$ & $p$ value \\
\hline Age, median (IQs) & $66(58-75)$ & $72(63-81)$ & $<0.01$ \\
\hline Female, $n(\%)$ & $53(38.7)$ & $49(44.1)$ & 0.75 \\
\hline Hypertension, $n$ (\%) & $104(75.9)$ & $90(81.1)$ & 0.33 \\
\hline Diabetes mellitus, $n$ (\%) & $35(25.6)$ & $35(31.5)$ & 0.30 \\
\hline Atrial fibrillation, $n(\%)$ & $40(29.2)$ & $31(27.9)$ & 0.83 \\
\hline Myocardial infarction, $n$ (\%) & $17(12.4)$ & $17(15.3)$ & 0.51 \\
\hline Previous stroke, $n(\%)$ & $14(10.2)$ & $16(14.4)$ & 0.31 \\
\hline Current smoking, $n(\%)$ & $37(27.0)$ & $27(24.3)$ & 0.63 \\
\hline NIHSS score on admission, median (IQs) & $6(4-13)$ & $15(7-19)$ & $<0.01$ \\
\hline Thrombolysis, $n$ (\%) & $77(56.2)$ & $61(55.0)$ & 0.84 \\
\hline Thrombectomy, n (\%) & $39(28.5)$ & $26(23.4)$ & 0.37 \\
\hline Etiology & & & 0.17 \\
\hline Large vessel disease, $n(\%)$ & $29(21.2)$ & $37(33.3)$ & \\
\hline Small vessel disease, $n(\%)$ & $9(6.6)$ & $4(3.6)$ & \\
\hline Cardioembolic, $n(\%)$ & $44(32.1)$ & $29(26.1)$ & \\
\hline Undetermined, $n(\%)$ & $49(35.8)$ & $39(35.1)$ & \\
\hline Other, $n(\%)$ & $6(4.4)$ & $2(1.8)$ & \\
\hline \multicolumn{4}{|l|}{ Cytokines } \\
\hline \multicolumn{4}{|l|}{ Ex vivo stimulation } \\
\hline IL-12p70, pg/ml, median (IQs) & $5.6(1.7-11.0)$ & $3.0(0.0-5.2)$ & $<0.01$ \\
\hline IL-10, pg/ml, median (IQs) & $44.4(32.0-69.3)$ & $65.6(40.4-89.4)$ & $<0.01$ \\
\hline IL-6, pg/ml, median (IQs) & 11611 (8279-16298) & $11534(8013-17757)$ & 0.96 \\
\hline IL-1 $\beta$, pg/ml, median (IQs) & $1611(1101-2313)$ & $1323(797-2095)$ & 0.01 \\
\hline IL-8, pg/ml, median (IQs) & $1528(953-2276)$ & $2256(1237-3459)$ & $<0.01$ \\
\hline TNFa, pg/ml, median (IQs) & $2570(1785-3793)$ & $2071(1518-2835)$ & $<0.01$ \\
\hline IP-10, pg/ml, median (IQs) & $474(25-832)$ & $288(138-534)$ & $<0.01$ \\
\hline \multicolumn{4}{|l|}{ Plasma } \\
\hline IL-6, pg/ml, median (IQs) & $3.2(1.9-6.5)$ & $8.9(4.6-25.2)$ & $<0.01$ \\
\hline
\end{tabular}

IQs interquartiles, NIHSS National Institutes of Health Stroke Scale

performance (Additional file 1: Figure S1). Cluster characteristics are available in the Additional file 2: Table S1.

In the unsupervised, blinded to outcome analysis, we obtained three clusters with the different pattern of cytokine concentrations (Fig. 1). Cluster $1(n=68)$ had relatively high release of IL-12p70, TNF $\alpha$, IP-10, IL-6, and IL-1 $\beta$; low synthesis of IL-10 and IL-8; and low level of plasma IL-6. Cluster $2(n=65)$ demonstrated relatively high release of IL-10, IL-8, IL-1 $\beta$, and IL-6; low release of IL-12p70, TNFa, and IP-10; and elevated level of plasma IL-6. Cluster $3(n=115)$ showed relatively low release of all cytokines after blood stimulation accompanied by the low level of plasma IL-6.

Kruskal-Wallis test followed by post-hoc characterization supported our visual observations (Additional file 2: Table S1). Cluster 1 had the highest release of IL-12p70 $(p<$
$0.01)$, TNF $\alpha(p<0.01)$, and IP-10 $(p<0.01)$ among all clusters. Cluster 2 had higher release of IL-10 $(p<0.01)$, IL-8 $(p<0.01)$, and plasma level of IL-6 $(p<0.01)$ compared to other clusters. Cluster 3 was characterized by the lowest release of IL-6 $(p<0.01)$, IL-1 $\beta(p<0.01)$, and TNF $\alpha(p<$ $0.01)$ among clusters. There were no differences between cluster 1 and 3 in the plasma level of IL-6 $(p=1.00)$ and release of IL-10 $(p=0.37)$ and IL-8 $(p=0.98)$.

The 3-month functional outcome differed between three clusters. We observed the lowest rate of poor outcome in cluster 1 , the highest in cluster 2 , and intermediate in cluster $3(25 \%, 69.2 \%$, and $42.6 \%$, respectively). When we used cluster 1 as a reference, the risk of poor outcome was increased 4.79-times $(95 \% \mathrm{CI} 2.13-10.78, p<0.01)$ for cluster 2 and 2.14-times (95\%CI 1.05-4.37, $p=0.04)$ for cluster 3 in the analysis adjusted for age and stroke severity. 
Table 2 Predictors of poor outcome identified in the univariate analysis and results of backward elimination models

\begin{tabular}{|c|c|c|c|c|c|c|c|c|c|}
\hline & \multicolumn{3}{|c|}{ Univariate analysis } & \multicolumn{3}{|c|}{$\begin{array}{l}\text { Multivariate analysis of individual cytokines adjusted } \\
\text { for age and stroke severity }\end{array}$} & \multicolumn{3}{|c|}{$\begin{array}{l}\text { Multivariate analysis of all cytokines adjusted for } \\
\text { age and stroke severity }\end{array}$} \\
\hline & $\mathrm{OR}$ & $95 \% \mathrm{Cl}$ & $p$ value & $\mathrm{OR}$ & $95 \% \mathrm{Cl}$ & $p$ value & OR & $95 \% \mathrm{Cl}$ & $p$ value \\
\hline Age & 1.04 & $1.02-1.06$ & $<0.01$ & 1.04 & $1.02-1.06$ & $<0.01$ & 1.03 & $1.01-1.06$ & 0.01 \\
\hline NIHSS on admission & 1.12 & $1.07-1.16$ & $<0.01$ & 1.12 & $1.07-1.17$ & $<0.01$ & 1.09 & $1.04-1.14$ & $<0.01$ \\
\hline \multicolumn{10}{|l|}{ Cytokines* } \\
\hline \multicolumn{10}{|l|}{ Ex vivo stimulation } \\
\hline IL-12p70 & 0.46 & $0.31-0.67$ & $<0.01$ & 0.50 & $0.34-0.74$ & $<0.01$ & 0.55 & $0.36-0.84$ & $<0.01$ \\
\hline $\mid \mathrm{L}-10$ & 1.55 & $1.17-2.05$ & $<0.01$ & 1.49 & $1.11-2.00$ & $<0.01$ & 1.83 & $1.32-2.56$ & $<0.01$ \\
\hline $\mathrm{IL}-1 \beta$ & 0.69 & $0.52-0.90$ & $<0.01$ & 0.74 & $0.55-0.99$ & 0.04 & 0.85 & $0.60-1.20$ & 0.36 \\
\hline IL-8 & 1.75 & $1.22-2.51$ & $<0.01$ & 1.54 & $1.06-2.22$ & 0.02 & 1.28 & $0.80-2.05$ & 0.31 \\
\hline TNFa & 0.62 & $0.46-0.84$ & $<0.01$ & 0.67 & $0.49-0.92$ & 0.01 & 0.64 & $0.44-0.92$ & 0.02 \\
\hline IP-10 & 0.61 & $0.44-0.84$ & $<0.01$ & 0.65 & $0.47-0.90$ & 0.01 & 0.89 & $0.59-1.34$ & 0.58 \\
\hline \multicolumn{10}{|l|}{ Plasma } \\
\hline IL-6 & 2.68 & $1.64-4.39$ & $<0.01$ & 1.86 & $1.17-2.94$ & $<0.01$ & 1.59 & $1.02-2.47$ & 0.04 \\
\hline
\end{tabular}

${ }^{*}$ Cytokines were standardized to the same scale (mean $=0 ; \mathrm{SD}=1$ )

NIHSS - National Institutes of Health Stroke Scale; OR- odds ratio; $95 \% \mathrm{Cl}-95 \%$ confidence interval

\section{Multimarker score}

To construct multimarker score, we used four cytokines that remained significantly associated with poor outcome in the final backward elimination model: IL-12p70, IL-10, TNF $\alpha$, and plasma IL-6. Table 3 shows the $\beta$ coefficients of included cytokines and predictive values of multimarker score.

In the multivariate analysis adjusted for age and stroke severity, patients with higher multimarker score had 2.72times per unit increased risk of worse outcome (95\% CI 1.86-3.99, $p<0.01)$. After addition of multimarker score to the best-fit clinical model, we observed improvement in discrimination of poor outcome (Fig. 2), supported by the increase in AUC $(p=0.02)$ and IDI $(p<0.01)$ (Table 3$)$. The model with the multimarker score was wellcalibrated (Hosmer-Lemeshow $\chi^{2} p=0.98$ ). Multimarker score also performed better than each of cytokines added to the best-fit clinical model $(p<0.05)$ : IL-12p70 (AUC = 0.768), IL-10 (AUC = 0.750), IL-8 (AUC = 0.746), TNF $\alpha$ $(\mathrm{AUC}=0.750)$, IP-10 $(\mathrm{AUC}=0.750)$, IL-1 $\beta(\mathrm{AUC}=$ 0.741), and plasma IL-6 (AUC = 0.752).

In addition, multimarker score significantly improved the classification accuracy for poor outcome compared to the best-fit clinical model as evidenced by categorical

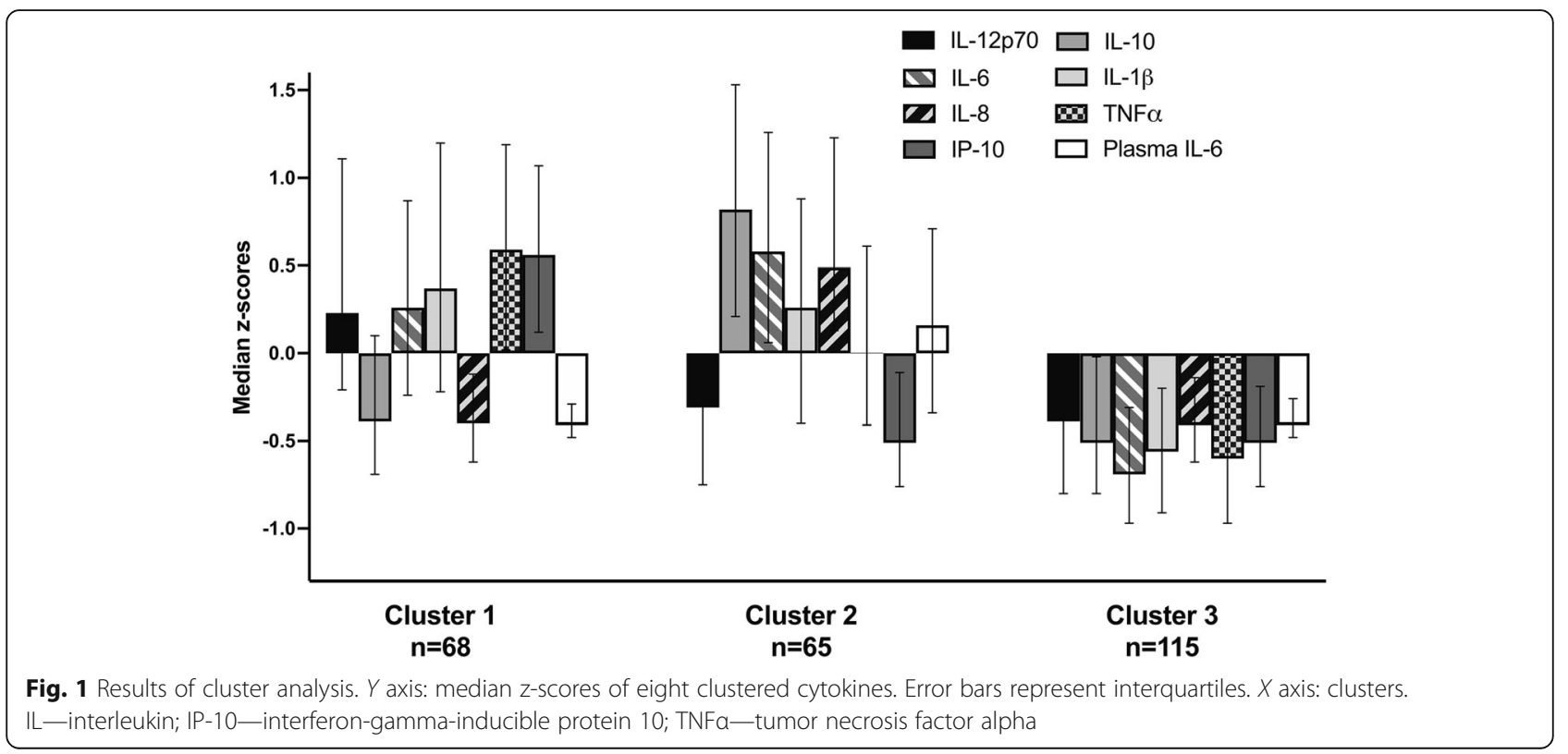


Table 3 Multimarker score and prediction of poor outcome

\begin{tabular}{|c|c|c|}
\hline & Poor outcome & $p$ value \\
\hline \multicolumn{3}{|l|}{ Cytokines included in the score* } \\
\hline IL-12p70, Coef $(95 \% C l)$ & $-0.60(-1.03$ to -0.17$)$ & $<0.01$ \\
\hline IL-10, Coef $(95 \% C l)$ & $0.61(0.27-0.94)$ & $<0.01$ \\
\hline TNFa, Coef $(95 \% \mathrm{Cl})$ & $-0.45(-0.82$ to -0.08$)$ & 0.02 \\
\hline Plasma IL-6, Coef $(95 \% C l)$ & $0.46(0.02-0.90)$ & 0.04 \\
\hline \multicolumn{3}{|l|}{ Score, per 1- unit increment } \\
\hline Unadjusted OR (95\%Cl) & $3.24(2.22-4.73)$ & $<0.01$ \\
\hline Adjusted + OR $(95 \% \mathrm{Cl})$ & $2.72(1.86-3.99)$ & $<0.01$ \\
\hline \multicolumn{3}{|l|}{ c Statistics } \\
\hline Best-fit clinical model, AUC (95\%Cl) & $0.731(0.668-0.794)$ & 0.02 \\
\hline Best-fit clinical model + multimarker score, AUC $(95 \% \mathrm{Cl})$ & $0.808(0.755-0.861)$ & \\
\hline IDI $(95 \% \mathrm{Cl})$ & $0.125(0.084-0.167)$ & $<0.01$ \\
\hline Continuous NRI (95\%Cl) & $0.672(0.437-0.907)$ & $<0.01$ \\
\hline Categorical NRI (95\%Cl) & $0.249(0.122-0.375)$ & $<0.01$ \\
\hline
\end{tabular}

$I D I$ integrated discrimination improvement, $N R I$ net reclassification improvement

*Levels of cytokines were standardized to the same scale (mean $=0, S D=1$ )

tAnalysis adjusted for age and stroke severity on admission

NRI $(0.249, p<0.01)$ and continuous NRI $(0.672, p<$ 0.01). Reclassification tables are shown in Additional file 3: Table S2. Among patients with poor outcome, $18.0 \%$ $(n=20)$ were correctly upclassified versus $6.3 \%(n=7)$ incorrectly downclassified. In the good outcome group $21.9 \%(n=30)$ were correctly downclassified whereas $8.8 \%(n=12)$ were incorrectly upclassified by multimarker score.

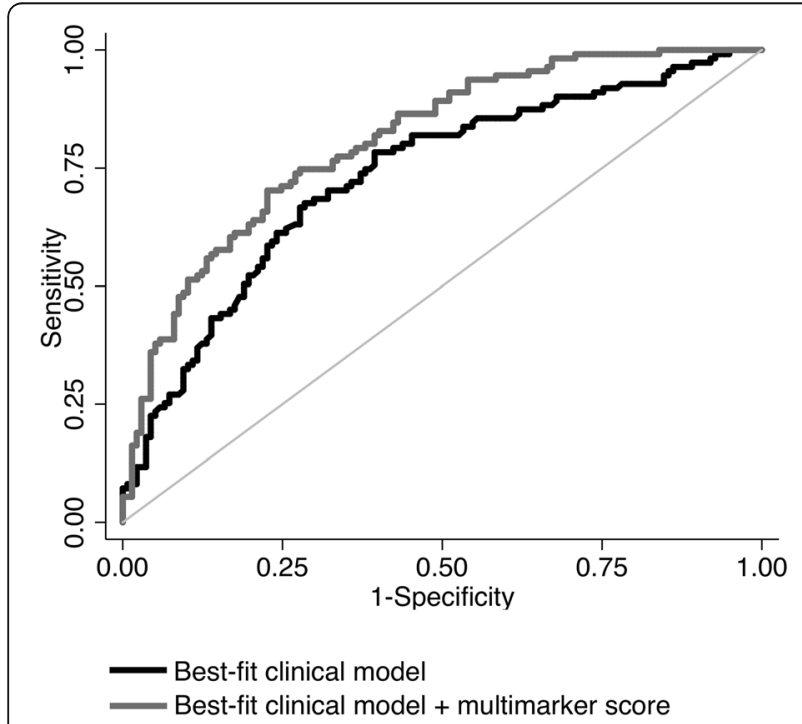

Fig. 2 Receiver operating characteristics. Black line represents the performance of the best-fit clinical model to discriminate patients with poor outcome. Gray line shows the performance of the enhanced model after addition of multimarker score

\section{Discussion}

Our study showed that ex vivo cytokine synthesis after whole blood stimulation with LPS differs between stroke patients with good outcome and patients with poor outcome. We found that the decreased release of TNF $\alpha$, IL$1 \beta$, IP-10, and IL-12 and the increased release of IL-10 and IL-8 are associated with poor functional outcome.

Emsley et al. studied ex vivo cytokine synthesis in 36 acute ischemic stroke patients [8]. They found the decreased TNF $\alpha$, IL-1 $\beta$, and IL- 6 production after in vitro blood stimulation in non-survivors and patients with poor functional outcome. Recently, we reported that the reduced release of TNF $\alpha$ and IP-10 is independently from age and stroke severity associated with worse prognosis after stroke [9].

The current study provides a new piece of information about a relationship between ex vivo synthesized cytokines and stroke outcome. It demonstrates the decreased production of IL-12 and increased release of IL-10 and IL-8 in patients with unfavorable prognosis. Interleukin12 is a pro-inflammatory cytokine that plays a key role in resistance to infections by modulating the activity of natural killer cells and $\mathrm{T}$ cells [25]. Interleukin-10 is a prototypic anti-inflammatory cytokine that limits the immune response to pathogens [26]. In stroke patients, higher plasma level of IL-10 is associated with the increased risk of infections [27] and lack of response to preventive antibacterial therapy [28]. Thus, the decreased IL-12 and increased IL-10 release from blood cells might contribute to post-stroke immunodepression.

Interleukin-8 is a chemokine involved in the recruitment and activation of neutrophils [29]. Neutrophils play 
an important role in ischemic brain injury [30]. Excessive neutrophil activation by IL-8 could contribute to greater brain damage after stroke and consequently leads to worse outcome.

Consistently with previous reports, we also observed the elevated level of circulating IL- 6 in patients with poor outcome $[6,7]$.

We did not find any association between ex vivo synthesized IL-6 and functional outcome. Emsley et al. reported the correlation between the reduced IL- 6 production and worse outcome after stroke [8]. This discrepancy between studies could be explained by several methodological differences including the sample size, the method of blood stimulation, and statistical approach. First, Emsley et al. measured cytokine production at several timepoints in the first week after stroke. Only minimal cytokine production was used to assess a correlation between cytokine synthesis and outcome. Second, Emsley et al. used much higher dose of LPS to stimulate blood cells $(200 \mathrm{ng} / \mathrm{mL}$ vs $10 \mathrm{ng} / \mathrm{mL})$. Third, the sample size differs between these two studies (36 vs 248 patients). Finally, we dichotomized outcome score and performed uni- and multivariate logistic regression analysis, whereas Emsley et al. correlated cytokine level with the mRS score.

Cluster analysis revealed interactions between synthesized cytokines, plasma IL-6, and stroke outcome. We identified three groups of patients with distinct cytokine profiles. The group with the worst outcome demonstrated relatively high synthesis of IL-10, IL-8, IL-1 $1 \beta$, and IL-6 and low synthesis of IL-12, IP-10, and TNF $\alpha$ accompanied by high circulating IL-6 level. The group with the best prognosis showed relatively high synthesis of TNF $\alpha$, IP-10, IL-12, IL-1 $\beta$, and IL-6 together with low synthesis of IL-10 and IL-8. Plasma level of IL- 6 was relatively low in this group. Patients with intermediate outcome had relatively low synthesis of all cytokines accompanied by low circulating IL-6. Our findings suggest that stroke outcome may depend on the interplay between stroke-induced immunodepression and systemic inflammation. The relatively low production of proinflammatory cytokines was associated with comparatively good outcome if immunodepression was not accompanied by systemic inflammation (low circulating IL-6). In experimental models of stroke, systemic inflammation is linked to poor outcome via permanent disruption of the blood-brain barrier, neutrophil mobilization and infiltration into the ischemic brain, impaired reperfusion, and enhanced platelet activity [10]. Immunodepression may worsen outcome by increasing the incidence of systemic infections [1]. Our cluster analysis also underlines the association between ex vivo IL-10 and IL-8 production and outcome. In contrast to patients with the best and intermediate prognosis, patients with the worst outcome had high synthesis of these cytokines.
Although observational studies are not able to demonstrate a causative relationship between cytokine synthesis and stroke outcome, both systemic inflammation and immunodepression could be considered as a potential therapeutic target. Agents that target components of IL6 signaling pathway (e.g., monoclonal antibodies against IL-6 or IL-6 receptor or Janus kinases inhibitors) might attenuate systemic inflammation [31]. In healthy men volunteers who received endotoxin intravenously, immunodepression was partially reversed by subcutaneous administration of interferon- $\gamma$ [32]. This treatment increased ex vivo LPS-induced TNF $\alpha$ release. In vitro $\beta$ glucan, a component of Candida albicans, partially reversed the LPS-induced immunodepression [33]. Ex vivo $\beta$-glucan treatment of monocytes from volunteers receiving LPS injection re-instanced their capacity for cytokine synthesis [31]. Of note, it remains unclear if reversion of post-stroke immunodepression will result in better outcome.

The accurate prediction of outcome after stroke is important in clinical practice. Biomarkers may facilitate stroke prognosis. Whiteley and colleagues proposed desirable features of a biomarker for stroke prognosis: (1) independent association of with outcome, (2) statistically significant predictive value added to a clinical model, and (3) improvement of the patients' classification [22].

Blood-derived inflammatory biomarkers might provide useful information to improve prediction of outcome after stroke [34]. In the second part of our study, we assessed the prognostic value of cytokine-based multimarker score. Our multimarker score included ex vivo synthesized IL-12, IL-10, TNF $\alpha$, and plasma IL-6. This multimarker score was independently associated with outcome after adjusting for the two most important stroke prognosticators: age and stroke severity. The addition of this score to the clinical model improved discrimination and reclassification. The multimarker score provided greater improvement in outcome prediction than individual cytokines. Although direct comparison between studies is difficult due to different methodologies, our multimarker score combining synthetized cytokines and plasma IL- 6 seems to have better predictive value than other inflammatory markers including circulating IL-6, C-reactive protein, or fibrinogen $[6,7,22$, $35]$. Given our results, it would be interesting to investigate in future studies the predictive ability of combination of ex vivo synthesized cytokines with other circulating inflammatory markers such as C-reactive protein or fibrinogen.

We need to address several limitations of our study. First, our results need confirmation in an independent population. Second, we performed whole blood stimulation 3 days after stroke. The prognostic value of our biomarkers measured early after stroke onset needs to be determined. 
Third, there are no widely acceptable thresholds to calculate category-based NRI. Similar to previous studies [7, 35], we applied stringent thresholds which are relevant for stroke clinical practice. Less rigorous thresholds could provide different results. Therefore, an optimal value of thresholds should be further tested in future studies.

We are aware that our novel biomarker is not ready for use in daily clinical practice. The necessity of blood culture in appropriate laboratory conditions strongly limits the technical feasibility of our biomarker. However, commercially available tubes with cell culture media and immune stimulant may offer a practical solution to monitoring cytokine synthesis without specialized technical experience [36]. They allow performing blood stimulation in room air using a heating block. Minimal sample handling and low amount of blood needed for stimulation are other advantages which may facilitate wider use of whole blood stimulation in clinics. It would be interesting to assess in future studies the combination of the cytokine-based composite biomarker with other non-inflammatory biomarkers (e.g., copeptin).

\section{Conclusions}

In conclusion, the decreased release of some proinflammatory cytokines (IP-10, TNF $\alpha$, IL-1 $\beta$, and IL12p70) and increased release of IL-10 and IL-8 after ex vivo blood stimulation with LPS are related to poor outcome after stroke. This association is modulated by plasma IL-6. Cytokine-based multimarker score composed of ex vivo released IL-12, IL-10, TNF $\alpha$, and plasma IL-6 adds prognostic value to clinical model for predicting stroke outcome.

\section{Supplementary information}

Supplementary information accompanies this paper at https://doi.org/10. 1186/s12974-019-1691-1.

Additional file 1: Figure S1. NbClust's optimal number of clusters. Among all indices provided by NbClust package in $\mathrm{R}, 12$ indices proposed 3 as the best number of clusters

Additional file 2: Table S1. Comparison of baseline characteristics and levels of cytokines between clusters

Additional file 3: Table S2. Reclassification table for poor outcome

\section{Abbreviations}

AUC: Under receiver operator curvesIDIIntegrated discrimination improvementILInterleukinIP-10Interferon-gamma-inducible protein 10IQsInterquartilesmRSModified Rankin ScaleNIHSSNational Institute of Health Stroke ScaleNRINet reclassification improvementTNFaTumor necrosis factor alpha

\section{Acknowledgements}

Not applicable

\section{Authors' contributions}

EKM prepared the study protocol; collected, analyzed, and interpreted the data; and prepared the manuscript. MP and JP collected and interpreted the data. KW performed the cytometric assay and analyzed the data. AS and MS revised the manuscript for intellectual content. TD prepared the study protocol; analyzed and interpreted the data; supervised the study; and prepared the manuscript. All authors read and approved the final manuscript.

\section{Funding}

This study was supported by the grant from the National Science Center (2015/19/B/NZ4/00287).

\section{Availability of data and materials \\ The datasets used during the current study are available from the corresponding author on reasonable request.}

Ethics approval and consent to participate

Written informed consent was obtained from each patient included in the study. The study protocol was approved by the Bioethics Committee of Jagiellonian University.

Consent for publication

Not applicable

\section{Competing interests}

The authors declare that they have no competing interests.

\section{Author details}

'Department of Neurology, Jagiellonian University Medical College, ul. Botaniczna 3, 31-503 Kraków, Poland. Department of Molecular Neuropharmacology, Maj Institute of Pharmacology, Polish Academy of Sciences, ul. Smetna 12, 31-343 Krakow, Poland. ${ }^{3}$ Department of Clinical Immunology, Institute of Paediatrics, Jagiellonian University Medical College, ul. Wielicka 265, 30-663 Krakow, Poland.

Received: 2 October 2019 Accepted: 24 December 2019

Published online: 06 January 2020

References

1. ladecola C, Anrather J. The immunology of stroke: from mechanisms to translation. Nat Med. 2011;17:796-808.

2. Chamorro Á, Meisel A, Planas AM, Urra X, van de Beek D, Veltkamp R. The immunology of acute stroke. Nat Rev Neurol. 2012 Jun 5;8:401-10.

3. Haeusler KG, Schmidt WUH, Föhring F, Meisel C, Helms T, Jungehulsing GJ, et al. Cellular immunodepression preceding infectious complications after acute ischemic stroke in humans. Cerebrovasc Dis. 2008;25:50-8.

4. Hug A, Dalpke A, Wieczorek N, Giese T, Lorenz A, Auffarth G, et al. Infarct volume is a major determiner of post-stroke immune cell function and susceptibility to infection. Stroke. 2009:40:3226-32.

5. Dziedzic T. Clinical significance of acute phase reaction in stroke patients. Front Biosci. 2008;13:2922-7.

6. Bustamante A, Sobrino T, Giralt D, García-Berrocoso T, Llombart V, Ugarriza I, et al. Prognostic value of blood interleukin- 6 in the prediction of functional outcome after stroke: a systematic review and meta-analysis. J Neuroimmunol. 2014:274:215-24.

7. Whiteley W, Jackson C, Lewis S, Lowe G, Rumley A, Sandercock P, et al, Inflammatory markers and poor outcome after stroke: a prospective cohort study and systematic review of interleukin-6. PLoS Med. 2009;6: e1000145.

8. Emsley HCA, Smith CJ, Gavin CM, Georgiou RF, Vail A, Barberan EM, et al. Clinical outcome following acute ischaemic stroke relates to both activation and autoregulatory inhibition of cytokine production. BMC Neurol. 2007;7:5.

9. Klimiec E, Kowalska K, Pasinska P, Pera J, Slowik A, Dziedzic T. Reduced release of TNFa and IP-10 after ex vivo blood stimulation with endotoxin is associated with poor outcome after stroke. Cytokine. 2018;102:51-4.

10. Dziedzic T. Systemic inflammation as a therapeutic target in acute ischemic stroke. Expert Rev Neurother. 2015;15:523-31.

11. Sacco RL, Kasner SE, Broderick JP, Caplan LR, Connors JJ, Culebras A, et al. An updated definition of stroke for the 21st century: a statement for healthcare professionals from the American Heart Association/American Stroke Association. Stroke. 2013;44:2064-89. 
12. Brott T, Adams HP, Olinger CP, Marler JR, Barsan WG, Biller J, et al. Measurements of acute cerebral infarction: a clinical examination scale. Stroke. 1989;20:864-70.

13. Adams HP Jr, Bendixen BH, Kappelle LJ, Biller J, Love BB, Gordon DL, et al. Classification of subtype of acute ischemic stroke. Definitions for use in a multicenter clinical trial. TOAST. Trial of Org 10172 in Acute Stroke Treatment. Stroke. 1993;24:35-41.

14. Rankin J. Cerebral vascular accidents in patients over the age of 60: II. Prognosis Scott Med J. 1957;2:200-15.

15. Kowalska K, Klimiec E, Weglarczyk K, Pera J, Slowik A, Siedlar M, et al. Reduced ex vivo release of pro-inflammatory cytokines and elevated plasma interleukin-6 are inflammatory signatures of post-stroke delirium. J Neuroinflammation. 2018:15:111.

16. Dormann CF, Elith J, Bacher S, Buchmann C, Carl G, Carre G, et al. Collinearity: a review of methods to deal with it and a simulation study evaluating their performance. Ecography. 2013;36:27-46.

17. Ward $\mathrm{H}$. Hierarchical grouping to optimize an objective function. J Am Stat Assoc. 1963;58:236-44.

18. Clatworthy J, Buick D, Hankins M, Weinman J, Horne R. The use and reporting of cluster analysis in health psychology: a review. $\mathrm{Br} J$ Health Psychol. 2005:10:329-58.

19. Charrad M, Ghazzali N, Boiteau V, Niknafs A. NbClust: an R package for determining the relevant number of clusters in a data set. J Stat Softw. 2014;61:1-36.

20. Rost NS, Bottle A, Lee JM, Randall M, Middleton S, Shaw L, et al. Stroke severity is a crucial predictor of outcome: an international prospective validation s. J Am Heart Assoc. 2016;5:e002433.

21. Pencina MJ, D'Agostino RB, Vasan RS. Evaluating the added predictive ability of a new marker: from area under the ROC curve to reclassification and beyond. Stat Med. 2008;27:157-72.

22. Whiteley W, Wardlaw J, Dennis M, Lowe G, Rumley A, Sattar N, et al. The use of blood biomarkers to predict poor outcome after acute transient ischemic attack or ischemic stroke. Stroke. 2012;43:86-91.

23. Pencina MJ, D'Agostino RB, Steyerberg EW. Extensions of net reclassification improvement calculations to measure usefulness of new biomarkers. Stat Med. 2011;30:11-21.

24. Kundu S, Aulchenko YS, van Duijn CM, Janssens ACJW. PredictABEL: an R package for the assessment of risk prediction models. Eur J Epidemiol. 2011; 26:261-4.

25. Trinchieri G. Proinflammatory and immunoregulatory functions of interleukin-12. Int Rev Immunol. 1998;16:365-96.

26. Fiorentino DF, Zlotnik A, Mosmann TR, Howard M, O'Garra A. IL-10 inhibits cytokine production by activated macrophages. J Immunol. 1991;147:381522.

27. Chamorro A, Amaro S, Vargas M, Obach V, Cervera A, Torres F, et al. Interleukin 10, monocytes and increased risk of early infection in ischaemic stroke. J Neurol Neurosurg Psychiatry. 2006;77:1279-81.

28. Klehmet J, Harms H, Richter M, Prass K, Volk HD, Dirnagl U, et al. Strokeinduced immunodepression and post-stroke infections: lessons from the preventive antibacterial therapy in stroke trial. Neuroscience. 2009;158:118493.

29. Baggiolini M, Clark-Lewis I. Interleukin-8, a chemotactic and inflammatory cytokine. FEBS Lett. 1992;307:97-101.

30. Jickling GC, Liu D, Ander BP, Stamova B, Zhan X, Sharp FR. Targeting neutrophils in ischemic stroke: translational insights from experimental studies. J Cereb Blood Flow Metab. 2015;35:888-901.

31. Garbers C, Heink S, Korn T, Rose-John S. Interleukin-6: designing specific therapeutics for a complex cytokine. Nat Rev Drug Discov. 2018;17:395-412.

32. Leentjens J, Kox M, Koch RM, Preijers F, Joosten LA, van der Hoeven JG, et al. Reversal of immunoparalysis in humans in vivo: a double-blind, placebo-controlled, randomized pilot study. Am J Respir Crit Care Med. 2012;186:838-45.

33. Novakovic B, Habibi E, Wang SY, Arts RJW, Davar R, Megchelenbrink W, et al. $\beta$-Glucan reverses the epigenetic state of LPS-induced immunological tolerance. Cell. 2016;167:1354-1368.e14.

34. Ramiro L, Simats A, García-Berrocoso T, Montaner J. Inflammatory molecules might become both biomarkers and therapeutic targets for stroke management. Ther Adv Neurol Disord. 2018;11: 1756286418789340 .
35. Swarowska M, Ferens A, Pera J, Slowik A, Dziedzic T. Can prediction of functional outcome after stroke be improved by adding fibrinogen to prognostic model? J Stroke Cerebrovasc Dis. 2016;25:2752-5.

36. Duffy D, Rouilly V, Libri V, Hasan M, Beitz B, David M, et al. Functional analysis via standardized whole-blood stimulation systems defines the boundaries of a healthy immune response to complex stimuli. Immunity. 2014:40:436-50.

\section{Publisher's Note}

Springer Nature remains neutral with regard to jurisdictional claims in published maps and institutional affiliations.
Ready to submit your research? Choose BMC and benefit from:

- fast, convenient online submission

- thorough peer review by experienced researchers in your field

- rapid publication on acceptance

- support for research data, including large and complex data types

- gold Open Access which fosters wider collaboration and increased citations

- maximum visibility for your research: over $100 \mathrm{M}$ website views per year

At BMC, research is always in progress.

Learn more biomedcentral.com/submissions 